\title{
SECONDARY CLASSES AND TRANSVERSE MEASURE THEORY OF A FOLIATION
}

\author{
BY S. HURDER AND A. KATOK
}

1. The purpose of this note is to announce several theorems showing how the secondary classes of a foliation $\mathcal{F}$ of a compact manifold $X$ depend upon the measure theoretic properties of the equivalence relation determined by the foliation. The relevant properties are:

(i) amenability [14], which is equivalent to hyperfiniteness by ConnesFeldman-Weiss [3]; and

(ii) the Murray-von Neumann type.

A set $B \subset X$ is saturated if it is the union of leaves of $₹$. The equivalence relation $₹$ has type $\mathrm{I}$ if there is a measurable subset of $X$ which intersects almost every leaf exactly once; type II if it admits an invariant measure, finite or infinite in the given measure class but does not have an essential saturated set of type I; and type III if it does not have any essential saturated sets of types I or II. Every equivalence relation can be decomposed into parts of types I, II, and III. These types correspond to certain algebraic properties of the von Neumann algebra $\mathcal{M}(X, \mathcal{F})$ associated with the equivalence relation $[\mathbf{1}$, 13].

Let $X$ be a compact manifold without boundary and $₹$ a $C^{2}$, codimension$n$ foliation of $X$. The secondary classes are given by a map $\Delta_{*}: H^{*}\left(\mathrm{WO}_{n}\right) \rightarrow$ $H^{*}(X ; \mathbf{R})$ with image spanned by the classes of the form $\Delta_{*}\left(y_{I} c_{J}\right)$. Here, $y_{I}$ is a basis element for the relative cohomology $H^{*}\left(\mathrm{gl}_{n}, \mathrm{O}_{n}\right)$, and $c_{J}$ is a Chern form of degree at most $2 n$. If degree $c_{J}=2 n$, we say the class is residual. The Godbillon-Vey classes are those of the form $\Delta_{*}\left(y_{1} c_{J}\right) \in H^{2 n+1}(X ; \mathbf{R})$, with $y_{1} \in H^{1}\left(\mathrm{gl}_{n}, \mathrm{O}_{n}\right)$, the normalized basis element. The generalized GodbillonVey classes are those of the form $\Delta_{*}\left(y_{1} y_{I} c_{J}\right)$, where $y_{I}=1$ is permitted. (For a convenient reference, see [11].)

The residual secondary classes have the unusual property that they localize to the measurable saturated subsets of $X$ : for each such $B \subset X$ and residual $y_{I} c_{J} \in H^{p}\left(\mathrm{WO}_{n}\right)$, the restriction $\Delta_{*}\left(y_{I} c_{J}\right) \mid B \in H^{p}(X)$ is well defined [5]. The following theorems are stated for the secondary classes of $₹$ on $X$, but corresponding theorems also hold for the localized classes $\Delta_{*}\left(y_{I} c_{J}\right) \mid B$ of the restriction $\mathcal{F} \mid B$.

THEOREM 1. If $\mp$ has type $\mathrm{I}$, then all residual secondary classes of $₹$ are zero.

Since $₹$ has type $I$ if and only if it is a fibration in the category of measurable equivalence relations, Theorem 1 generalizes the well-known fact that the secondary classes are zero for a smooth fibration.

Received by the editors March 21, 1984 and, in revised form, July 2, 1984.

1980 Mathematics Subject Classification. Primary 46L10, 57R30, 58F11.

(C)1984 American Mathematical Society $0273-0979 / 84 \$ 1.00+\$ .25$ per page 
THEOREM 2. Assume there is a generalized $G-V$ class which is nonzero for $\mathcal{F}$. Then $\mathcal{F}$ contains a nontrivial component of type III. If $\mathcal{F}$ is ergodic, $\mathcal{M}(X, \mathcal{F})$ must be a type III factor.

The factors of type III are divided into subtypes $\mathrm{III}_{0}, \mathrm{III}_{\lambda}$ for $0<\lambda<1$ and $\mathrm{III}_{1}$. An ergodic foliation of type $\mathrm{III}_{\lambda}$ has an absolutely continuous transverse measure which is multiplied by powers of $\lambda$ under the holonomy maps. If this measure is given by a $C^{1}$ density, it is easy to see that the G-V class $\Delta_{*}\left(y_{1} c_{1}^{n}\right)$ is zero [6]. One can speculate whether this holds for all type $\mathrm{III}_{\lambda}$-foliations and what role the type III classification plays in the values of the G-V classes. For a recent development in the case of type $\mathrm{III}_{0}$ foliations, see Connes [2].

THEOREM 3. If $\mathcal{F}$ is amenable then all residual secondary classes $\Delta_{*}\left(y_{I} c_{J}\right)$ $\in H^{p+2 n}(X ; \mathbf{R})$ for $p>1$ are zero.

The original Rousarrie examples of foliations with $\Delta_{*}\left(y_{1} c_{1}^{n}\right)$ nonzero are amenable, so Theorem 3 cannot be extended to the secondary classes of degree $2 n+1$ without additional hypotheses. This is discussed further in [6].

If almost every leaf of $\mathcal{F}$ has subexponential growth, then $\mathcal{F}$ is amenable. Combining Theorem 3 with Theorem 1 of [6] yields:

COROLLARY 4. If almost every leaf of $₹$ has subexponential growth, then all residual secondary classes of $\mathcal{F}$ are zero.

2. Given a generalized G-V class $y_{I} c_{J} \in H^{*}\left(\mathrm{WO}_{n}\right)$, using Thurston's realization theorems and the topological methods of $[\mathbf{7 , 9}$ ] it is often possible to construct on a given compact orientable manifold $X$ uncountably many distinct codimension- $n$ foliations for which $\Delta_{*}\left(y_{I} c_{J}\right)$ takes distinct nonzero values. The above theorems then place restrictions on the possible dynamics of such $\mathcal{F}$ and the measure properties of its von Neumann algebra $\mathcal{M}(X, \mathcal{F})$. Our general expectation is that the residual secondary classes of $\mathcal{F}$ directly define invariants of $\mathcal{M}(X, \mathcal{F})$ and the $C$-algebra $C:(X, \mathcal{F})$ (cf. [2]) and for $y_{I} c_{J}$ with $y_{I} \in H^{*}\left(\mathrm{sl}_{n}, \mathrm{O}_{n}\right)$ we conjecture these classes are invariants of orbit equivalence.

3. Sketch of proofs. For each cohomology class $y_{I} \in H^{*}\left(\mathrm{gl}_{n}, \mathrm{O}_{n}\right)$ there is a Weil measure $\chi\left(y_{I}\right)$ on the quotient measure space $X / \mathcal{F}$. These generalize the Godbillon measure $g=\chi\left(-2 \pi \cdot y_{1}\right)$ introduced by Duminy to study the G-V class of codimension one foliations [4]. The residual secondary classes of $\mathcal{F}$ are determined by the values of the Weil measures, so it suffices to show the appropriate $\chi\left(y_{I}\right)=0$. Theorems 1-3 are consequences of our study of how the measures $\chi\left(y_{I}\right)$ depend on the transverse dynamics of $\mathcal{F}$. Let $Q \rightarrow X$ denote the normal bundle to $\mathcal{F}$. The following is an essential tool for calculating the Weil measures.

THEOREM 5.' Let $h_{0} \mid$ be a $\mid$ measurable $\mid$ metric on $|Q \rightarrow X|$ whose restriction to each leaf of $\mathcal{F}$ is smooth. If $h_{0}$ has a uniform bound on all of $X$ for the norms of the first derivatives of $h_{0}$ in leaf directions, then the Weil measure

\footnotetext{
${ }^{1}$ This result was announced by the first author at the AMS Special Session on Foliations in East Lansing, Michigan, November 1982.
} 
$\chi\left(y_{I}\right)$ can be calculated from the measurable family of closed forms $\left\{\chi\left(y_{I}\right) \mid L\right.$ s.t. $L$ is a leaf of $\mathcal{F}\}$ defined leafwise by means of the smooth metrics $\left\{h_{0} \mid L\right.$ s.t. $L$ is a leaf of $F\}$.

To prove Theorem 1 we use the assumption that $\mathcal{F}$ has a measurable crosssection to show there is a metric $h_{0}$ on $Q$ satisfying Theorem 5 and which is almost everywhere holonomy invariant. This implies the restricted classes $\chi\left(y_{I}\right) \mid L$ vanish a.e., so the measures $\chi\left(y_{I}\right)=0$ and the theorem follows.

For Theorem 2 assume there is an absolutely continuous invariant transverse measure for $₹$ with almost every leaf essential. Then Theorem 5 implies the Godbillon measure $g=0$ and all classes $\Delta_{*}\left(y_{1} y_{I} c_{J}\right)=0$, a contradiction.

For Theorem 3 we use a fundamental theorem of Zimmer [14] to conclude that the normal smooth $\mathrm{GL}_{n}(\mathbf{R})$-cocycle of an amenable foliation is measurably equivalent to a cocycle taking values in one of $2^{n}$ maximal amenable subgroups of $\mathrm{GL}_{n}(\mathbf{R})$. The key result is to show this cocycle can be made tempered or locally bounded in leaf directions so that Theorem 5 applies. This follows from some general results concerning cocycles over metric equivalence relations with values in linear Lie groups. More specifically, let $\mathcal{F}$ be a discrete equivalence relation provided with a measurable family of metrics on the leaves so that any ball contains finitely many elements and the number of those elements grow at most exponentially with the radius. We call such an object a metric equivalence relation of exponential type.

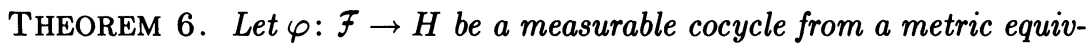
alence relation of exponential type into a maximal amenable subgroup $H$ of $\mathrm{GL}_{n}(\mathbf{R})$. If $\varphi$ is measurably equivalent to a tempered cocycle with values in $\mathrm{GL}_{n}(\mathbf{R})$, it is also measurably equivalent to a tempered cocycle with values in $H$.

This is one of the series of results concerning the asymptotic behavior and tempering of cocycles over group actions and metric equivalence relations. Other results will appear in $[\mathbf{1 0}, \mathbf{1 2}]$.

We then use a result of $[8]$ to conclude that for $y_{I}$ of degree $p>1$, the class $\Delta_{*}\left(y_{I}\right) \mid L$ is exact for a.e. leaf $L$, and there is a global measurable bounded $(p-1)$-form on $X$ implementing this. By the leafwise Stokes' Theorem [5] and Theorem 5 , the Weil measure $\chi\left(y_{I}\right)$ is zero.

Full proofs of the above plus related results will appear in [10].

Our techniques suggest the following:

CONJECTURE 7. For $y_{I} \in H^{*}\left(\mathrm{sl}_{n}, \mathrm{O}_{n}\right)$, the Weil measure $\chi\left(y_{I}\right)$ is quasiinvariant under orbit equivalence: If $(X, \mathcal{F})$ and $\left(X^{\prime}, \mathcal{F}^{\prime}\right)$ are orbit equivalent, then $\chi\left(y_{I}\right)$ assigns the value zero to corresponding sets in $X / \mathcal{F}$ and $X^{\prime} / \mathcal{F}^{\prime}$.

\section{REFERENCES}

1. A. Connes, Sur la integration non-commutative, Lecture Notes in Math., vol. 725, SpringerVerlag, 1979, pp. 19-143..

2. - Cyclic cohomology and the transverse fundamental class of a foliation (preprint).

3. A. Connes, J. Feldman and B. Weiss, An amenable equivalence relation is generated by a single transformation, Ergodic Theory Dynamical Systems 1 (1981), 431-450. 
4. G. Duminy, L'invariant de Godbillon-Vey d'un feuilletage se localise dans les feuilles ressort, 1982 (preprint).

5. J. Heitsch and S. Hurder, Secondary classes, Weil measures and the geometry of foliations, J. Differential Geometry (to appear).

6. S. Hurder, The Godbillon measure of amenable foliations, 1984 (preprint).

7. _ - On the classifying space of smooth foliations, Illinois J. Math. (to appear).

8. ___ Foliation dymamics and leaf invariants, Comment. Math. Helv. (to appear).

9. - Characteristic classes of flat Diff X-bundles, 1984 (preprint).

10. S. Hurder and A. Katok, Ergodic theory and Weil measures of foliations, 1983 (preprint).

11. F. Kamber and $\mathrm{Ph}$. Tondeur, Foliated bundles and characteristic classes, Lectures Notes in Math., vol. 493, Springer-Verlag, 1975.

12. A Katok, Ergodic theorems and $\varepsilon$-classification of cocycles over dymamical systems (in preparation).

13. C. C. Moore, Ergodic theory and von Neumann algebras, Proc. Sympos. Pure Math., part 2, Amer. Math. Soc., Providence, R.I., 1982, pp. 179-226.

14. R. Zimmer, Induced and amenable ergodic actions of Lie groups, Ann. Sci. École Norm. Sup. 11 (1978), 407-428.

MATHEMATICAL SCIENCES RESEARCH INSTITUTE, 2223 FUlton STREeT, BERKELEY, CALIFORNIA 94720 